CERN-PPE/97-79

13.6.1997

\title{
QUADRUPOLE MOMENTS AND MEAN SQUARE CHARGE RADII IN THE BISMUTH ISOTOPE CHAIN
}

J. Kilgallon ${ }^{1}$, M.R. Pearson ${ }^{1}$, J. Billowes ${ }^{1}$, P. Campbell ${ }^{1}$, U. Georg ${ }^{2}$, I.S. Grant ${ }^{1}$, M. Keim ${ }^{2}$, R. Neugart ${ }^{2}$, M. Neuroth ${ }^{2}$, S.Wilbert ${ }^{2}$ and the ISOLDE Collaboration ${ }^{3}$

${ }^{1}$ Schuster Laboratory, University of Manchester, M13 9PL, UK.

${ }^{2}$ Institut für Physik, Universität Mainz, D-55099 Mainz, Germany

${ }^{3}$ CERN, CH-1211 Geneva 23, Switzerland

\begin{abstract}
Isotope shifts and hyperfine structures of the ${ }^{205,206,208,210,210 m, 212,213} \mathrm{Bi}$ isotopes have been studied on the $306.7 \mathrm{~nm}$ line using gas cell laser spectroscopy. The neutronrich isotopes are the first isotones of $\mathrm{Pb}$ to be measured immediately above the $\mathrm{N}=126$ shell closure. The ground state quadrupole moments of the even- $\mathrm{N}$ isotopes increase as neutrons are added or removed from the $\mathrm{N}=126$ shell, but no corresponding increase is observed in the charge radii.
\end{abstract}

IS344

(Accepted for publication in Physics Letters B) 
Isotope shifts and nuclear ground state moments have been measured for bismuth isotopes across the $\mathrm{N}=126$ shell closure. This is the first time that isotope shifts of a neighbour of a closed proton shell isotope chain (in this case, the ${ }_{82} \mathrm{~Pb}$ chain) have been measured across a neutron shell closure. A careful comparison with the $\mathrm{Pb}$ isotones sheds new light on the way the Bi nuclear charge distributions produce their quadrupole moments. Information on the charge distribution is provided both by the spectroscopic quadrupole moment $\mathrm{Q}_{s}$ and by the isotope shift which depends on the change in the mean square charge (msc) radius $\delta\left\langle r^{2}\right\rangle$ between two isotopes. A common approximation to describe the increased size due to a quadrupole shape deformation is

$$
\left\langle r^{2}\right\rangle=\left\langle r^{2}\right\rangle_{0}\left(1+\frac{5}{4 \pi} \beta_{2}^{2}\right)
$$

where $\beta_{2}$ is the quadrupole deformation parameter and $\left\langle r^{2}\right\rangle_{0}$ is the msc radius of the spherical nucleus. In most isotope chains, a small deformation-component in the msc radius can be masked by shell dependent volume effects in $\left\langle r^{2}\right\rangle_{0}$ as neutrons are added or removed. In the case of the bismuth isotopes, a much greater sensitivity to deformation effects is obtained by comparing relative changes in $\left\langle r^{2}\right\rangle$ with the neighbouring (spherical) lead isotopes. In this way it is possible to demonstrate that the quadrupole core polarization by the $h_{9 / 2}$ proton does not produce any corresponding increase in charge radii.

The quadrupole moment systematics of non-collective states in the vicinity of the doubly-magic nucleus ${ }^{208} \mathrm{~Pb}$ have been interpreted as single-particle alignments which induce additional core deformations $[1,2]$. The core stiffness is reduced as neutrons are removed from the $\mathrm{N}=126$ major shell and systematic quadrupole moment enhancements are seen in a range of prolate and oblate nuclear configurations [1, 3]. The work reported here on the neutron-rich bismuth isotopes, coupled with previous results for the neutrondeficient isotopes [4] show that the concept of a simple core shape change is inconsistent with the measured msc radii. Such shapes would give rise to a change in msc radius outside experimental tolerance. In contrast however, the spherical shell model can give a reasonable account of the quadrupole moments and qualitatively explain the absence of any effect on the radii.

The $A=205,206,208,210,210 \mathrm{~m}, 212$ and 213 bismuth isotopes were measured at the ISOLDE facility at CERN using the technique of resonant laser fluorescence spectroscopy of atoms in an inert buffer gas; a technique which has been described by Sprouse et al [5]. This work represents the first laser spectroscopic measurements of the $A=210,210 \mathrm{~m}, 212$ and 213 isotopes that lie beyond the $\mathrm{N}=126$ shell closure.

The radioisotopes were produced by proton spallation of uranium and thorium targets at a beam energy of $1 \mathrm{GeV}$. Samples of the $A=205,206,212$ and 213 bismuth isotopes were obtained as the granddaughters in the decay chains of francium isotopes produced from a uranium target with a thermal ion source and tungsten surface ioniser. Samples of the $A=208,210$ and $210 \mathrm{~m}$ isotopes were produced by the spallation of thorium targets and extracted using a hot plasma-discharge ion source. In both cases the radioisotopes were implanted in separate on-line collections in $300 \mu \mathrm{g} \mathrm{cm}^{-2}$ lead coatings on tantalum foils. The bismuth sample sizes ranged from $10^{9}$ to $10^{11}$ atoms. The implanted foils were incorporated in a thermal oven which was inserted into a gas cell. The cell was evac- 
uated and filled with typically 9 Torr of argon. The radioactive bismuth samples were released as free atoms by electrical heating of the foils to evaporate the thin lead coatings off the tantalum. Laser spectroscopy was performed during the slow diffusion of the bismuth atoms through the cell, as described in refs $[4,6]$. The atoms were excited by frequency-doubled CW light pumping the ${ }^{4} \mathrm{~S}_{3 / 2}{ }^{4} \mathrm{P}_{1 / 2}$ transition at $306.7 \mathrm{~nm}$. Resonance fluorescence was detected on the $472 \mathrm{~nm}$ decay branch of the ${ }^{4} \mathrm{P}_{1 / 2}$ state to the ${ }^{2} \mathrm{D}_{3 / 2}$ first excited state. The experimental background was mostly due to the radioactivity of the samples but included some light from the hot foil. A typical spectrum is shown in figure 1. The frequency calibration for this spectrum was attained from the monitoring of the fundamental laser frequency's transmission through a $1.5 \mathrm{GHz}$ FSR etalon recorded during the experimental scan.

Hyperfine coefficients were extracted from complete fits to the observed structure. Absolute frequency positions were determined relative to components arising from stable ${ }^{209} \mathrm{Bi}$. The appearance of ${ }^{209} \mathrm{Bi}$ in each spectrum was controlled by the careful selection of high purity lead for the catcher material with each coating containing between $10^{9}$ and $10^{10}$ atoms of the stable isotope. The extracted isotope shifts, hyperfine factors and moments are presented in table 1 . The magnetic moments of the radioactive bismuth isotopes were deduced by scaling from the known ${ }^{209} \mathrm{Bi}$ moment assuming no hyperfine anomaly. A value of $4.1106(2) \mu_{N}[7]$ was taken for the magnetic moment of ${ }^{209} \mathrm{Bi}$. The spectroscopic quadrupole moments were determined in a similar fashion using $\mathrm{Q}_{s}(209)=-0.370(26) \mathrm{b}$, the weighted average of two muonic X-ray experiments [7]. The magnetic moment for ${ }^{206} \mathrm{Bi}$ is significantly different from an earlier, apparently erroneous measurement [8]. The new magnetic moment systematics will be discussed in a fuller report of this work [9].

Key results for bulk parameters, $\mathrm{Q}_{s}$ and $\delta\left\langle r^{2}\right\rangle$, derived from the new Bi measurements are presented in figures 2 and 3. Following the example of Mahnke [1], who used plots of $\mathrm{Q}_{s}^{N} / \mathrm{Q}_{s}^{N=126}$ as a function of neutron number to illustrate the trends in nuclear quadrupole polarization, figure 2 shows the relative quadrupole moments of these bismuth ground states and those of high spin isomers with $\left(h_{9 / 2}\right)^{n}$ proton configurations, where $n=1,2,4$, and $\left(h_{9 / 2}\right)^{2} i_{13 / 2}$ valence proton configurations of At (the original data for these systems may be found in ref [1]). Figure 3 shows the relative isotope shifts for the $\mathrm{Bi}$ (this work and ref [6]), Rn [10], Fr [11] and Ra [12] isotope chains compared to those of $\mathrm{Pb}$ [13], normalising to the $\mathrm{N}=124,126$ pair $\left(\delta \nu^{124,126}=+1\right)$ which has been measured in all chains.

The quadrupole moments for all isotope chains shown in figure 2 progressively increase with distance from the $\mathrm{N}=126$ shell closure. The absolute increase is proportional to the moment at $\mathrm{N}=126$ for a particular proton configuration. These nuclei, by virtue of their close proximity to the doubly magic shell closure, lend themselves to particle + core descriptions and the enhancements observed in the quadrupole moments have been attributed to a polarization of the core, the stiffness of which seems to depend on the neutron number alone [1,4]. An analysis of the isotope shifts can show whether this core polarization also affects the charge radii. The isotope shift is the difference in the frequency of a particular atomic transition in two isotopes. In heavy elements it is dominated by the field shift which is directly proportional to the change in the nuclear msc radius between the two isotopes, $\delta\left\langle r^{2}\right\rangle^{A A^{\prime}}$ [14]. The relative isotope shifts shown in figure 3 , to an excellent approximation, therefore reflect relative $\delta\left\langle r^{2}\right\rangle^{A A^{\prime}}$ changes in this region. 
Despite an extreme sensitivity to nuclear shape changes $[4,6,15,16]$ the relative radii in the $\mathrm{Bi}$ and $\mathrm{Pb}$ chains show very close correspondence across the $\mathrm{N}=126$ shell closure. In the particular case of the even- $\mathrm{N}$ Bi isotopes the average charge distributions show no discernible difference in relative size compared to their lead isotones.

Ground state quadrupole moments of the odd $\mathrm{Pb}$ isotopes [13] show no evidence of any developing core deformation. In the comparison of relative charge radii or isotope shifts (figure 3) any difference in the onset of deformation between the $\mathrm{Pb}$ chain and a neighbouring isotone system would result in a visible change in the course of the system's relative charge radii (with the more deformed system's data points lying upwardly shifted from those of $\mathrm{Pb}$ ). Such deformation changes are apparent in figure 3 for the light radons and heavy radon, francium and radium isotopes. The sensitivity of such comparisons to changes in static deformation can be estimated using the ${ }^{203} \mathrm{Rn}$ data as this is the first isotope to display clear departure and a sizeable quadrupole moment. The collective model can be invoked to relate the spectroscopic quadrupole moment, $\mathrm{Q}_{s}$, to an intrinsic deformation of the nuclear shape. In the strong coupling limit the intrinsic moment, $\mathrm{Q}_{0}$, is related via

$$
\mathrm{Q}_{s}=\frac{3 K^{2}-I(I+1)}{(I+1)(2 I+3)} \mathrm{Q}_{0}
$$

where $K$ is the projection of the nuclear spin on the intrinsic axis of symmetry and $\mathrm{Q}_{0} \approx\left(3 Z R_{0}^{2} / \sqrt{5 \pi}\right) \beta_{2}$ to first order in $\beta_{2}$ (here $R_{0}$ is conventionally taken to be the liquid drop radius, $1.2 \mathrm{~A}^{1 / 3} \mathrm{fm}$ ). In this limit, a core deformation of $\beta_{2} \simeq+0.06$ is deduced for ${ }^{203} \mathrm{Rn}$, once the small neutron single particle contribution has been subtracted. This deformation would produce an upward shift of the ${ }^{203} \mathrm{Rn}$ msc radius above $\mathrm{Pb}$ of +0.043 $\mathrm{fm}^{2}$ (using equation 1 ) or +0.4 in the normalized units of figure 3 . The observed deviation is approximately twice this estimate which suggests that dynamic effects or higher order deformations may also be contributing. There is no obvious inconsistency in applying a similar analysis to the neutron-rich Rn and Ra isotopes, although lack of quadrupole moment data and the additional effects of octupole deformations in this region preclude any clear conclusions. In the case of both ${ }^{203} \mathrm{Bi}$ and ${ }^{213} \mathrm{Bi}$ collective estimates of static deformation, similar to that performed for ${ }^{203} \mathrm{Rn}$, yield $\beta_{2} \sim-0.03$. The increase in msc radius this deformation would cause (equation 1) would be detectable in figure 3 and is incompatible with the almost perfect correspondence observed between the $\mathrm{Pb}$ and $\mathrm{Bi}$ radii.

Two essentially different nuclear models have been previously used in the study of quadrupole moments of aligned systems in the $\mathrm{Pb}$-region. The first, a deformed independent particle model $[17,18,19]$, utilizes a static core deformation and a Nilsson-type potential. Although this model has been successfully applied to the theoretical study of high spin isomers in the $\mathrm{Pb}$ region and has proved adequately capable of describing general features and electromagnetic properties (such as quadrupole moments) it predicts increases in msc radii that are not observed in the Bi isotopes. An alternative, the spherical shell model, requires no intrinsic core deformation. Relatively large quadrupole moments can be produced by the shell model if sufficient core polarization charges are present. Such modelling has been performed by Sagawa et al. [20] to calculate the quadrupole moments of the $\mathrm{N}=126$ high spin isomers. Core polarization charges induced by single particle states coupling to giant resonances were sufficient to reproduce the observed $\mathrm{Q}_{s}$ 
without any intrinsic core deformation. The quadrupole moments of the even-N bismuth ground states studied in this work are compared to a shell model calculation in table 2. The moments were calculated with the PBPOP interaction [21] restricting the proton to the $h_{9 / 2}$ orbit and allowing neutron particles in the $g_{9 / 2}$ and $i_{11 / 2}$ orbits and neutron holes in the $p_{1 / 2} p_{3 / 2}$ and $f_{5 / 2}$ orbits [22]. The effective charges for proton and neutrons were $e_{\pi}=+1.27 \mathrm{e}$ and $e_{\nu}=+0.9 \mathrm{e}$. Despite the necessary restrictions on the model space, the results are in good agreement with experiment.

A property of the spherical harmonic oscillator shell model of relevance here is that all states of the nucleus have similar rms charge and matter radii irrespective of the quadrupole moments, provided the only active orbits are from the same oscillator shell. An accurate illustration of the independence of the charge radius from the quadrupole moment for nuclei close to the shell closure is given by the ${ }^{210} \mathrm{Bi}$ ground state and isomer results in table 1 . The isomer shift between the $I=1$ ground state and $I=9$ isomer is zero within experimental errors. However the spectroscopic quadrupole moments of the two states differ substantially; $\mathrm{Q}_{s}(\mathrm{I}=1)=-0.29(3) \mathrm{Q}_{s}(\mathrm{I}=9)$. In a shell model framework, both states arise from the same valence configuration, $\left(\pi \mathrm{h}_{9 / 2}\right)\left(\nu \mathrm{g}_{9 / 2}\right)$, and a relationship between their shell model quadrupole moments can be readily formed. Using standard jj angular coupling reduction identities,

$$
Q_{s}(I=1) / Q_{s}(I=9)=-\frac{4}{15} \quad(=-0.267),
$$

which in comparison to the experimental ratio, $-0.29(3)$, clearly demonstrates the descriptive power of such modelling. The two systems may be viewed as almost identical except for the orbit orientations. The reorientation of the valence orbitals from equatorial to polar paths gives rise to a change in quadrupole moment but no change in msc radius and no isomer shift.

The shell model is therefore able to account for the quadrupole moment systematics in the bismuth isotopes and qualitatively explain the absence of any deformation effect in the charge radii. The radii would be expected to show an effect only when particle-hole excitations became significant, leading to an increase in the occupation of orbits with larger rms radius.

A full shell model treatment of nuclear electromagnetic properties close to ${ }^{208} \mathrm{~Pb}$ is a formidable task. Such modelling in the $\mathrm{Pb}$ chain [13] produced reasonable results for even-even $\mathrm{Pb}$ isotopes but wholly failed to reproduce the observed odd-even staggering of the charge radii seen in figure 3 (although some odd-even variation was produced by the calculations). The magnitude of the experimental odd-even staggering in the isotope chains discussed above is actually greater than the shifts evaluated for strongly coupled core deformations. A proper description of the core polarization influence on charge radii will not be possible until the mechanisms controlling the odd-even staggering are adequately understood.

The authors would like to thank Professor Alex Brown for helpful advice and performing the shell model calculations. 


\section{References}

[1] H.-E. Mahnke, Hyp. Int. 34 (1987) 47 and H.-E. Mahnke et al Phys. Lett. 122B (1983) 27.

[2] G. Scheveneels et al Phys. Rev. C43 (1991) 2560.

[3] G. Neyens et al The Proc. of the International Conference on Nuclear Shapes and Nuclear Structure at Low Excitation Energies, June 20-25 1994, Editions Frontiers.

[4] P. Campbell et al Nucl. Phys. A598 (1996) 61.

[5] G.D. Sprouse et al Phys. Rev. Lett. 63 (1989) 1463.

[6] P. Campbell et al Phys. Lett. B346 (1995) 21.

[7] P. Raghavan At. Data Nuc. Data Tables 42 (1989) 189.

[8] I. Lindgren and C.M. Johansson Arkiv. Fysik 15 (1959) 445.

[9] M. Pearson et al to appear.

[10] W. Borchers et al Hyp. Int. 34 (1987) 25.

[11] S.A. Ahmad et al Nucl. Phys. A483 (1988) 244.

[12] A. Coc et al Nucl. Phys. A468 (1987) 1.

[13] R.C. Thompson et al J. Phys. G9 (1983) 443.

[14] W.H. King Isotope shift in atomic spectra (1984) Plenum Press, London.

[15] D. Kowalewska et al Phys. Rev. A44 (1991) R1442.

[16] J. Billowes and P. Campbell J. Phys. G21 (1995) 707.

[17] G. Andersson et al Nucl. Phys. A268 (1976) 205.

[18] K. Matsuyanagi et al Nucl. Phys. A307 (1978) 253.

[19] K. Neergard et al Phys. Lett. B99 (1981) 191.

[20] H. Sagawa and A. Arima Phys. Lett. B202 (1988) 15.

[21] N.A.F.M. Poppelier and P.W.M Glaudemans Z. Phys. A329 (1988) 275.

[22] B.A. Brown Private communication.

[23] E. Browne \& R. B. Firestone Table of Radioactive Isotopes (1986) John Wiley \& Sons, New York.

[24] M. Barboza-Flores et al Z. Phys. A321 (1985) 85.

[25] R.S. Title and K.F. Smith Phil. Mag. 5 (1960) 1281.

[26] S.S. Albert et al Phys. Rev. 125 (1962) 256. 


\begin{tabular}{||c|c|c|c|c|c|c||}
\hline $\begin{array}{c}\text { Mass } \\
\text { and Spin[23] }\end{array}$ & $\mathrm{A}\left({ }^{4} \mathrm{~S}_{3 / 2}\right)$ & $\mathrm{MHz}\left({ }^{4} \mathrm{~S}_{3 / 2}\right)$ & $\mathrm{A}\left({ }^{4} \mathrm{P}_{1 / 2}\right)$ & Isotope Shift & $\mu$ & $\mathrm{Q}$ \\
\hline $202, \mathrm{I}=5 \dagger$ & $-405(10)$ & $-592(48)$ & $4590(14)$ & $-10267(58)$ & $4.259(14)$ & $-0.720(77)$ \\
\hline $203, \mathrm{I}=9 / 2$ & $-433(6)$ & $-549(40)$ & $4810(15)$ & $-8303(54)$ & $4.017(13)$ & $-0.667(68)$ \\
\hline $204, \mathrm{I}=6$ & $-332(32)$ & $-400(120)$ & $3881(13)$ & $-7681(85)$ & $4.322(15)$ & $-0.49(15)$ \\
\hline $205, \mathrm{I}=9 / 2$ & $-437.0(1.0)$ & $-481(10)$ & $4867(6)$ & $-5632(21)$ & $4.065(7)$ & $-0.585(43)$ \\
\hline $206, \mathrm{I}=6$ & $-356.1(1.5)$ & $-318(20)$ & $3916(6)$ & $-4926(23)$ & $4.361(8)$ & $-0.387(36)$ \\
\hline $207, \mathrm{I}=9 / 2$ & - & $-480(90)^{[a]}$ & $4887(9)^{[a]}$ & $-2995(60)^{[a]}$ & $4.081(9)^{[d]}$ & $-0.58(12)^{[d]}$ \\
\hline $208, \mathrm{I}=5$ & $-462(5)$ & $-522(38)$ & $4993(9)$ & $-1578(17)$ & $4.633(10)$ & $-0.635(64)$ \\
\hline $209, \mathrm{I}=9 / 2$ & $-446.97(4)^{[b]}$ & $-304.3(8)^{[b]}$ & $4921.9(5.4)^{[b]}$ & 0 & $4.1106(2)^{[d]}$ & $-0.370(26)^{[d]}$ \\
\hline $210, \mathrm{I}=1$ & $21.78(3)^{[c]}$ & $112.38(3)^{[c]}$ & - & $2495(85)$ & $-0.04451(6)^{[d]}$ & $+0.137(10)^{[d]}$ \\
\hline $210 \mathrm{~m}, \mathrm{I}=9$ & $-147(6)$ & $-387(40)$ & $1633(25)$ & $2463(42)$ & $2.728(42)$ & $-0.471(59)$ \\
\hline $212, \mathrm{I}=1$ & $-156(19)$ & $80(225)$ & $1727(207)$ & $7761(104)$ & $0.321(38)$ & $+0.10(28)$ \\
\hline $213, \mathrm{I}=9 / 2$ & $-339.3(1.8)$ & $-491(25)$ & $4449(7)$ & $10507(24)$ & $3.716(7)$ & $-0.597(52)$ \\
\hline
\end{tabular}

Table 1: Hyperfine coupling parameters extracted for the studied bismuth isotopes. All results taken from this work and that of Ref. [4] except [a] Ref. [21], [b] Refs. [21,22], [c] Ref. [23] and [d] Ref. [7]. † reevaluation for a possible spin of $\mathrm{I}=6$ is offered in Ref [4].

\begin{tabular}{||c||c|c||}
\hline Mass & $\begin{array}{c}\mathrm{Q}_{s} \text { (barns) } \\
\text { experimental }\end{array}$ & $\begin{array}{c}\mathrm{Q}_{s} \text { (barns) } \\
\text { shell model }\end{array}$ \\
\hline 203 & $-0.67(7)$ & -0.68 \\
\hline 205 & $-0.59(4)$ & -0.66 \\
\hline 207 & $-0.58(12)$ & -0.65 \\
\hline 209 & $-0.37(3)$ & -0.36 \\
\hline 211 & - & -0.49 \\
\hline 213 & $-0.60(5)$ & -0.52 \\
\hline
\end{tabular}

Table 2: Experimental and shell model predictions [19] of the ground state quadrupole moment of the even-N Bi isotopes. 


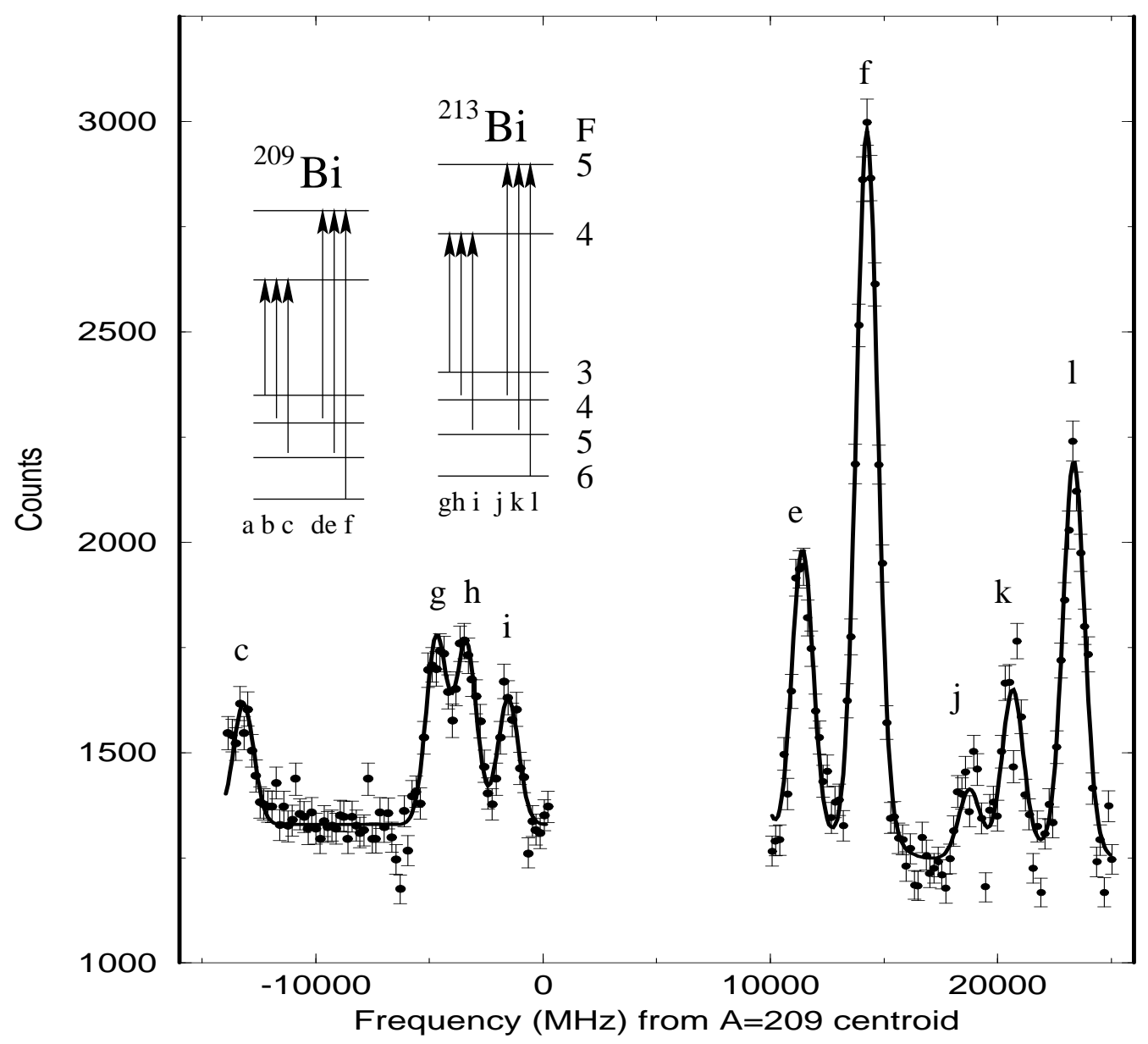

Figure 1: A spectrum of a ${ }^{213} \mathrm{Bi}$ sample in the presence of stable ${ }^{209} \mathrm{Bi}$ contamination. The position of hyperfine components associated with each isotope is as labelled. A full multi-component Voigt profile fit to the data is also shown. 


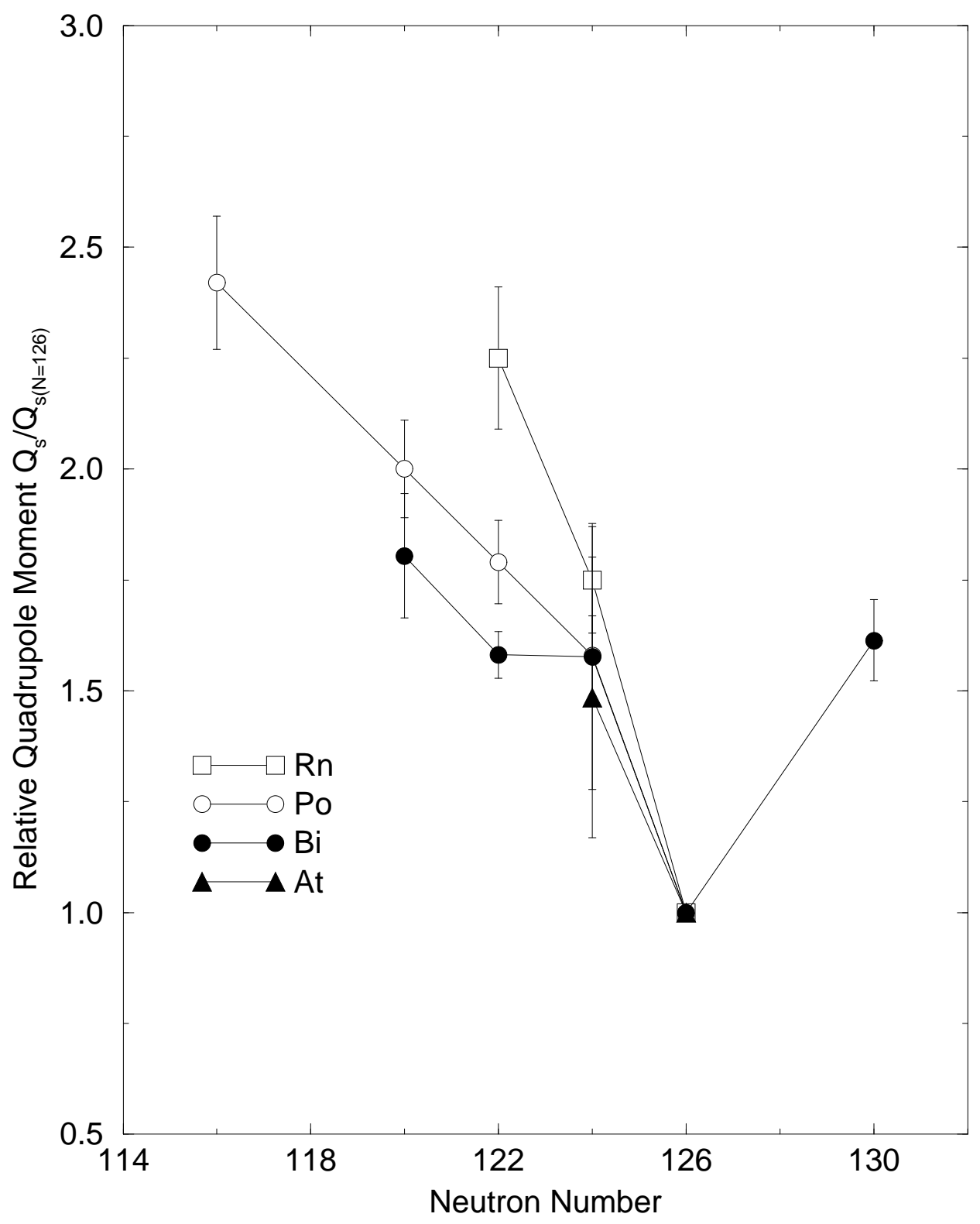

Figure 2: The relative quadrupole moments, $\mathrm{Q}_{s}^{N} / \mathrm{Q}_{s}^{N=126}$, of even-N bismuth ground states and high spin isomers in the region above $\mathrm{Z}=82$. 


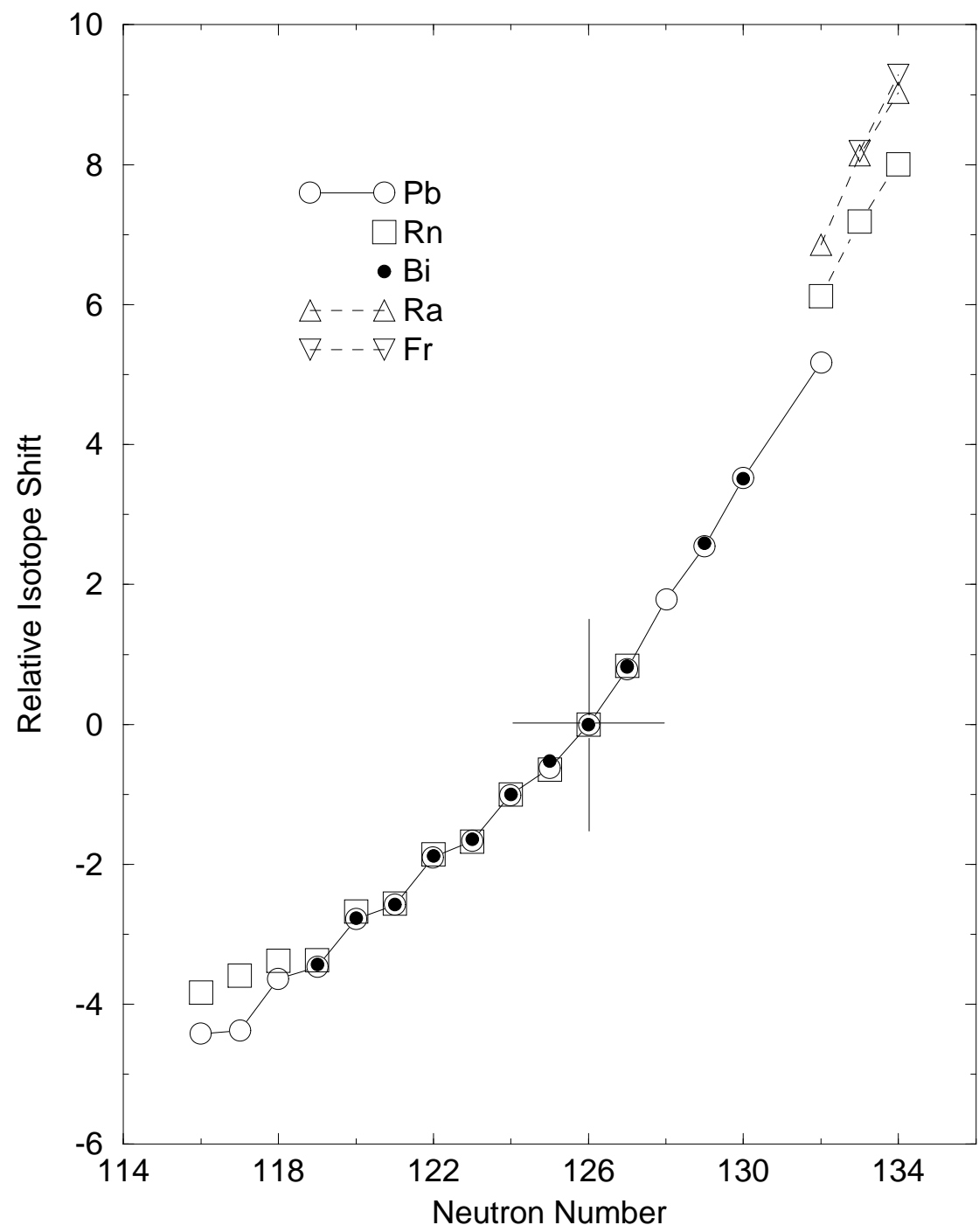

Figure 3: Comparison of the $\mathrm{Pb}, \mathrm{Bi}, \mathrm{Rn}, \mathrm{Fr}$ and Ra relative isotope shifts with each chain normalized to $\mathrm{IS}\left(\mathrm{N}=124, \mathrm{~N}^{\prime}=126\right)=1$. 